tischen Gleichungen als Anwendung der Eigenschaften des größten gemeinsamen Teilers behandelt und geometrisch veranschaulicht. - Unter einigen nebensächlichen Punkten, mit denen der Referent nicht ganz einverstanden ist, möchte er auf die Definition des Differentialquotienten hinweisen, die dessen Quotientenform als ein bloßes Symbol bezeichnet, während man $a f(x)$ einfach als die Funktion $f^{\prime}(x)$. $h$ zweier Unabhängigen: $x$ und $h$ erklären kann, woraus bei der Annahme: $f(x)=x$ die Gleichheiten $d x=h$ und $d f(x)=f^{\prime}(x) d x$ ohne jede Symbolik folgen. - Eine Ausgestaltung dieser Arbeit in Buchform wäre sehr erwünscht; in dieser müßten wohl noch die gebrochene lineare Funktion und einige andere elementare algebraische und transzendente Funktionen sowie die einfachsten Gleichungen zwischen zwei Veränderlichen erscheinen; zur Unterscheidung der unendlich Kleinen verschiedener Ordnung wären Beispiele aus der Physik aufzunehmen.

F.

\title{
Lehr- und Übungsbuch der Geometrie für Untergymnasien.
} Von F. Hočevar. 8. Auflage, 122 S. Wien, F. Tempsky, 1907.

Das Lehrbuch bat sich im Unterricht bewährt. Der Referent hält eine Vermehrung der Aufgabensammlung für erwünscht sowie einige kleinere Änderungen: z. B. sollte die Teilung der Strecken nicht erst bei der Ähnlichkeitslehre, sondern so früh wie möglich angegeben werden; vor dem Satz von Cavalie $\mathrm{r}$ i sollte dje Inhaltsgleichheit gerad er Prismen mit gleichen Höhen and Grundfächen durch Zerlegung nachgewiesen werden.

$F$.

Geometrische Transformationen. Von Dr. Karl D o e h l e man n, Professor an der Universität München. II. Teil. Die quadratischen und höheren birationalen Punkttransformationen. Mit 84 Figuren. Leipzig, G. J. Göschensche Verlagshandlung 1908. (Sammlung Schubert XXVIII) (VIII + 328 S.).

Die Bedeutung, welche die Transformationen für die verschiedenen Gebiete der Geometrie gewonnen haben, isł kaum zu überschätzen. Man wird nicht fehlgehen, wenn man einen überaus wesentlichen, vielleicht den wesentlichsten Unterschied zwischen der älteren und neueren Geometrie in der großen Rolle erblickt, welche innerhalb dieser letzteren den geometrischen Verwandtschaften zukommt.

Der Verfasser hat es im vorliegenden 2. Bande seines Werkes unternommen, eine Einleitung in die Theorie der höheren birationalen Transformationen zu geben, nachdem er im 1. Bande die Theorie der projektiven Verwandtschaften behandelt hatte. Nicht darum handelt es sich ihm, möglichst viele geometrische Ergebnisse in den Kreis seiner Betrachtungen zu ziehen, sondern das von ihm erstrebte und erreichte Ziel ist, den sachgemäß abgegrenzten Stoff so darzubieten, daß die leitenden Gedanken zur Geltung kommen und doch an die Fassungskraft der Studierenden keine zu hohen Ansprüche gestellt werden. Schwierigere Betrachtungen sind vermieden und durch blofe Hinweise auf ihre Ergebnisse ersetzt.

Das Buch beginnt mit einer Theorie der quadratischen Verwandtschaft der Ebene. Der ausgezeichnete Spezialfall der Transformation durch reziproke Radien wird besonders behandelt und auch die Mechanismen zur Herstellung einer solchen Verwandtschaft erörtert, nachdem als Anwendung die anallegmatische Erzeugung der Bizirkularkurven vierter Ordnung herangezogen 